\title{
Comparative Performance Evaluation of Routine Malaria Diagnosis at Ho Municipal Hospital
}

\author{
James Osei-Yeboah, ${ }^{1}$ Gameli Kwame Norgbe, ${ }^{2}$ Sylvester Yao Lokpo, ${ }^{3}$ \\ Mohammed Khadijah Kinansua, ${ }^{1}$ Loverage Nettey, ${ }^{3}$ and Emmanuel Alote Allotey ${ }^{1}$ \\ ${ }^{1}$ Department of Medical Laboratory Sciences, School of Allied Health Sciences, University of Health and Allied Sciences, Ho, Ghana \\ ${ }^{2}$ School of Allied Health Sciences, University of Health and Allied Sciences, Ho, Ghana \\ ${ }^{3}$ Laboratory Department, Ho Municipal Hospital, Ghana Health Service, Ho, Volta Region, Ghana \\ Correspondence should be addressed to James Osei-Yeboah; joyeboah@uhas.edu.gh
}

Received 18 May 2016; Revised 10 August 2016; Accepted 24 August 2016

Academic Editor: José F. Silveira

Copyright (C) 2016 James Osei-Yeboah et al. This is an open access article distributed under the Creative Commons Attribution License, which permits unrestricted use, distribution, and reproduction in any medium, provided the original work is properly cited.

\begin{abstract}
Differences in quality performance score had been reported for the routinely used diagnostic methods for malaria at different settings. There is therefore a need to evaluate the test performance of the routine diagnostic methods for malaria detection in Ho, a setting with no recorded quality evaluation on malaria diagnosis. The hospital-based cross-sectional study was conducted comprising 299 outpatients. Patients were first seen and presumptively diagnosed with malaria by a clinician and were referred to the laboratory for confirmation (microscopy and Rapid Diagnostic Test). The performance analysis included sensitivity, specificity, receiver operating characteristics (ROC), weighted kappa, Youden index, and $p$ value. Out of the 299 patients, 221 patients were positive by presumptive diagnosis, 35 were positive by Rapid Diagnostic Test (RDT), and 25 were positive by microscopy. Using microscopy as the gold standard, RDT had sensitivity of $62.5 \%$ and specificity of $92.73 \%$, whilst presumptive diagnosis had a sensitivity of $70.83 \%$ and specificity of $25.82 \%$. The RDT recorded ROC of 0.697 with $p$ value of 0.0001 . The presumptive diagnosis recorded ROC of 0.506 with $p$ value of 0.7304 . Though none of the test methods evaluated over the gold standard achieved the WHO recommended diagnostic sensitivity and specificity, the RDT achieved an acceptable agreement with the gold standard.
\end{abstract}

\section{Introduction}

Malaria continues to be a worldwide burden despite global efforts to curb the disease [1]. In Ghana, malaria is one of the main causes of adult morbidity and the leading cause of workdays loss to illness [2]. Malaria also accounts for $44 \%$ of outpatient attendance, $13 \%$ of all hospital deaths, and $22 \%$ of mortality among children less than five years of age [2]. The need for effective and practical diagnostic tests for global malaria control is increasing since effective diagnosis reduces both complications and mortality from malaria [3]. The lack of precise malaria diagnosis remains an obstacle to the treatment adherence [1]. Misdiagnosis of malaria will result in overdiagnosis, overprescription of antimalaria drugs, under diagnosis, and inappropriate treatment of nonmalaria febrile patients [4]. The World Health Organization recommends that every suspected malaria case should undergo prompt parasitological confirmation by microscopy or alternatively by Rapid Diagnostic Test [5]. Thus treatment solely on the basis of clinical suspicion should only be considered when a parasitological diagnosis is not available [5]. Clinical diagnosis of malaria is traditional among medical doctors and this method which is based on patients' signs and symptoms or on physical findings during examination is least expensive and most widely used [3]. Clinical diagnosis is widely used in areas where laboratory facilities are not available; however, it is unreliable due to the signs and symptoms of malaria being similar to other diseases [3]. Microscopy remains the gold standard for routine laboratory diagnosis of malaria, although it is not accessible and affordable in most peripheral health facilities [6]. Rapid Diagnostic Test (RDT), an immunochromatographic capture procedure, was developed to improve the timeless sensitivity and objectivity of malaria diagnosis through less reliance on expert microscopy [7]. 
Although RDTs clearly show promise as new diagnostic tool for Africa, it is not clear whether RDTs should replace presumptive therapy or light microscope nor is it clear which RDT is more appropriate for different epidemiological settings [8].

Despite an obvious need for improvement, malaria diagnosis is the most neglected area of malaria research [9]. Prompt and accurate diagnosis is critical to the effective management of malaria [3]. For an effective and timely treatment of malaria, the diagnostic method used should be accurate [9]. This will prevent misdiagnosis which can lead to drug misuse, increase in cost of antimalaria drugs, and also death of the patient [4]. It is estimated that a diagnostic test with $95 \%$ sensitivity and $95 \%$ specificity requiring minimal infrastructure would avert more than 100,000 deaths and about 400 million unnecessary treatments [10]. There is no known study carried out at the Ho Municipal Hospital that sought to assess the diagnostic efficiency of malaria in the facility. This study therefore seeks to comparatively evaluate the diagnosis efficiency of the various malaria diagnostic methods in the facility.

\section{Materials and Methods}

2.1. Study Design and Study Population. A purposive convenient cross-sectional study was carried out between January 2016 and April 2016 at the Ho Municipal Hospital in the Volta Region of Ghana. The study population is comprised of all outpatients who presented with signs and symptoms common to malaria infection (fever, bodily pains, headaches, chills, general weakness and loss of appetite, etc.). These patients, aged between five (5) months and eighty-five (85) years, were first seen, presumptively diagnosed with malaria by clinicians, and referred to the laboratory for confirmation (microscopy and Rapid Diagnostic Test). Participation was voluntary and patients who were excluded were those who were unwilling to participate, inpatients, patients reporting for review, and those with unrelated cause of ailment to malaria.

2.2. Sample Size Determination. Using the average monthly total malaria test requested (528) for two previous months (November 2015 and December 2015), a total study population of 2112 was generated for the four months study duration, using the Raosoft online sample size calculator (Raosoft. Inc, 2004). The recommended minimum sample of 289 participants was calculated at 95\% confidence level, 5\% margin of error, and a response distribution of $68 \%$ based on the average routine laboratory malaria positive test in the two previous months irrespective of the method used.

2.3. Blood Sample Collection. Using standard phlebotomy procedure, about $2 \mathrm{~mL}$ of venous blood was drawn and dispensed into ethylenediaminetetraacetic acid anticoagulant (EDTA) tubes by qualified technicians working in the hospital facility. The sample was then taken to the laboratory and used for the Rapid Diagnostic Test and field microscopy testing.
2.4. Rapid Diagnostic Test. Rapid Diagnostic Test was carried out as routinely done without any special attention given to the samples by biomedical scientist working in the municipal hospital laboratory. All Rapid Diagnostic Tests were done using Bioline SD malaria antigen Pf. manufactured by Standard Diagnostic, Inc, Korea. Assays were carried out as described by the manufacturer. In brief, all kit components and specimen were brought to room temperature prior to testing. Using a $5 \mu \mathrm{l}$ disposable capillary pipette, whole blood was drawn and transferred into the round sample well. Four (4) drops of assay diluent was added into the square assay diluent well holding the diluent bottle vertically. Reading of test was done in 15 minutes and for samples that tested negative repeated reading was done in 30 minutes. The presence of one colour band (" $\mathrm{C}$ " Control line) within the result window was interpreted as negative results. The presence of two colour bands ("T" Test line and " $\mathrm{C}$ " Control line) within the result window was interpreted as positive results. In the event where the control line fails to appear within the results window, the test was invalidated.

2.5. Microscopy. An amount of $6 \mu \mathrm{L}$ and $2 \mu \mathrm{L}$ of the blood sample was pipetted for the preparation of thick and thin blood films, respectively. Thin film was fixed with methanol for 5 minutes and both thin and thick film were stained with $10 \%$ Giemsa for 10 minutes. Stained slides were left to airdry before examination using a 100x objective oil immersion light microscope. The smears were independently read by two microscopists who were blinded to the results of the RDT as well as the diagnosis made by the clinicians and between each other. Parasites were counted against 200 white blood cells (WBCs) from the thick film. The parasite density was obtained by assuming a total WBC count of $8000 / \mathrm{mL}$ and at least 200 fields were examined before being taken as a negative result.

2.6. Data Analysis. The sensitivity and specificity of each of the three test methods were calculated by comparing to a composite reference gold standard generated from the three methods. The composite reference method was defined as a method that was positive for malaria parasites by all of the three methods (Presumptive, RDT, and Microscopy) and also negative for malaria parasites by all of the three methods. This gives the method $100 \%$ hypothetical sensitivity, specificity, and positive and negative predictive values [7].

Taking blood slide microscopy as the gold standard, the performance of the presumptive diagnosis method and the Rapid Diagnostic Test was evaluated to generate diagnostic accuracy summary statistics including receiver operative characteristics test, weighted kappa, $p$ values, and Youden J. statistics using MedCalc Version 14.2.0.0 for Windows (Vienna, Austria, https://www.medcalc.be/).

\section{Results}

Using a composite reference as gold standard which was generated from the three diagnostic methods, only 10 people were found to be truly positive for malaria and 65 people 
TABle 1: Performance of rapid, presumptive, and microscopic diagnostic test using the composite and microscopy as gold standards.

\begin{tabular}{lcccc}
\hline Parameter & Sensitivity & Specificity & PPV & NPV \\
\hline Composite as a standard & & & & \\
Rapid Diagnostic Test & 4.78 & 72.22 & 28.57 & 24.62 \\
Presumptive diagnosis & 43.48 & 23.55 & 4.52 & 83.33 \\
Field microscopy & 4.55 & 82.28 & 41.67 & 23.64 \\
Microscopy as a standard & & & & \\
Rapid Diagnostic Test & 62.50 & 92.73 & 42.86 & 96.59 \\
Presumptive diagnosis & 70.83 & 25.82 & 7.69 & 91.03 \\
\hline
\end{tabular}

Data is presented as percentages. PPV: positive predictive value and NPV: negative predictive value.

TABle 2: Age stratified performance of rapid and presumptive diagnostic test using field microscopy as a gold standard.

\begin{tabular}{lcccc}
\hline Parameter & Sensitivity & Specificity & PPV & NPV \\
\hline 55 years & & & & \\
$\quad$ Rapid Diagnostic Test & 85.71 & 86.89 & 42.86 & 98.15 \\
$\quad$ Presumptive diagnosis & 71.43 & 26.23 & 10.00 & 88.89 \\
6-18 years & & & & \\
$\quad$ Rapid Diagnostic Test & 30 & 100 & 100 & 85.11 \\
$\quad$ Presumptive diagnosis & 7.41 & 92.31 & 66.67 & 32.43 \\
19-40 years & & & & \\
$\quad$ Rapid Diagnostic Test & 37.5 & 96.88 & 50.00 & 94.90 \\
$\quad$ Presumptive diagnosis & 75 & 30.21 & 8.22 & 93.55 \\
41-85 years & & & & \\
$\quad$ Rapid Diagnostic Test & 50 & 97.18 & 60.00 & 95.83 \\
$\quad$ Presumptive diagnosis & 66.67 & 22.95 & 7.84 & 87.50 \\
\hline
\end{tabular}

Data is presented as percentages. PPV: positive predictive value and NPV: negative predictive value.

were found to be truly negative giving a hypothetical 100\% sensitivity and specificity. Evaluating each of the diagnostic methods over the composite reference, RDT reported a sensitivity of $4.78 \%$ and a specificity of $72 \%$. The Presumptive diagnosis method showed a sensitivity of $43.48 \%$ and a specificity of $23.55 \%$, while the field microscopy method had a sensitivity of $4.55 \%$ and a specificity of $82.28 \%$. Using field microscopy as the gold standard, RDT reported a sensitivity of $62.5 \%$ and a specificity of $92.73 \%$, while presumptive diagnosis reported a sensitivity of $70.83 \%$ and a specificity of $25.82 \%$. The presumptive diagnosis was found to have a low positive predictive value (7.69) against the field microscopy technique. See Table 1.

Comparing the two diagnostic methods to field microscopy in relation to the different age groups, RDT reported the highest sensitivity of $85.71 \%$ among $\leq 5$ years age group and specificity of $100 \%$ among the age group of 6-18 years. Using presumptive diagnosis, the highest sensitivity of $75 \%$ was reported among the age group of 19-40 years and a specificity of $92.31 \%$ was reported for the $6-18$ years age bracket. The presumptive diagnosis exhibited a low positive predictive value across the different age categorization; the exception though was found for the 6 to 18 years group. See Table 2.
TABLE 3: Interrater diagnostic performance criteria for rapid and presumptive diagnosis of malaria with field microscopy as a gold standard.

\begin{tabular}{|c|c|c|c|c|}
\hline Parameter & AUC (ROC) & $p$ value & Kappa & Youden \\
\hline \multicolumn{5}{|l|}{ Total population } \\
\hline Rapid Diagnostic Test & 0.697 & $<0.0001$ & 0.457 & 0.395 \\
\hline Presumptive diagnosis & 0.506 & 0.7304 & -0.007 & 0.013 \\
\hline \multicolumn{5}{|l|}{ Male participants } \\
\hline Rapid Diagnostic Test & 0.702 & 0.0035 & 0.492 & 0.404 \\
\hline Presumptive diagnosis & 0.504 & 0.8891 & 0.005 & 0.009 \\
\hline \multicolumn{5}{|l|}{ Female participants } \\
\hline Rapid Diagnostic Test & 0.695 & 0.0005 & 0.436 & 0.390 \\
\hline Presumptive diagnosis & 0.512 & 0.6156 & -0.013 & 0.023 \\
\hline
\end{tabular}

AUC: area under curve and ROC: receiver operative characteristic. Kappa $<0.20$ : poor, $0.41-0.60$ : moderate, $0.61-0.80$ : good, and $0.81-1$ : very good. $p$ is significant at 0.05 .

TABLE 4: Dose response threshold for rapid and presumptive diagnostic tests stratification by parasite density in thick blood smear.

\begin{tabular}{lccc}
\hline \multirow{2}{*}{ Parameter } & \multicolumn{3}{c}{ Parasitic count $(\mathrm{count} / \mu \mathrm{l})$} \\
& $1-100$ & $101-1000$ & $\geq 1000$ \\
\hline $\begin{array}{l}\text { Field microscopy } \\
\quad \text { Positive }\end{array}$ & $\mathbf{1}$ & $\mathbf{4}$ & $\mathbf{1 9}$ \\
$\begin{array}{l}\text { Rapid Diagnostic Test } \\
\quad \text { Positive }\end{array}$ & $1(100)$ & $3(75.00)$ & $11(57.89)$ \\
$\quad$ Negative & $0(0.00)$ & $1(25.00)$ & $8(42.11)$ \\
Presumptive diagnosis & & & \\
$\quad$ Positive & $0(0.00)$ & $4(100)$ & $13(68.42)$ \\
$\quad$ Negative & $1(100)$ & $0(0.00)$ & $6(31.58)$ \\
\hline
\end{tabular}

Data is presented as figures and percentages.

The quality of the test method as a medical diagnostic tool was assessed using the Youden J. index on a scale of $0-1$ as shown in Tables 3 and 4 . The performances of the RDT and presumptive diagnosis evaluated using field microscopy as the gold standard reported Youden J. index of 0.395 and 0.013 , respectively. The gender stratified performance evaluation revealed higher diagnostic ability for RDT method compared to the presumptive diagnosis method irrespective of the gender categorization.

Using the interrater agreement analysis, the weighted kappa was estimated to assess how results produced by RDT and presumptive diagnosis agree with field microscopy in the diagnosis of malaria. Agreement ranged from moderate (0.457) for RDT to poor $(-0.007)$ for presumptive diagnosis.

Assessing the effectiveness of the RDT and the presumptive diagnosis as methods for the diagnosis of malaria, the receiver operating characteristic (ROC) analysis was used to estimate the area under the diagnostic curve (AUC), ranging from 0.5 , a worthless test, to 1 , a perfect test. The RDT was shown to be a more effective test method (AUC $=0.697$; $p<0.0001)$ than the presumptive diagnosis $(\mathrm{AUC}=0.506$; $p=0.7304)$. As seen in Tables 3 and 4 , gender variations 
in performance were not prominent for both diagnostic methods (RDT and presumptive diagnosis). See Table 3.

Out of the 24 patients that were reported as having positive malaria parasite by the field microscopy method, 15 and 17 were positive for RDT and presumptive diagnosis, respectively (true positive). Among the 264 patients who tested negative for RDT, 9 were positive for field microscopy (false negatives). Among the 78 patients reported to be negative by presumptive diagnosis, 7 tested positive using the field microscopy technique (false negative). Among the false negative recording using the RDT $11.11 \%$ was within a parasitic count of $101-1000 / \mu \mathrm{l}$ and the rest (88.89\%) had a parasitic count of $1000 / \mu$ l or greater. Among the false negative recording using presumptive diagnosis $14.29 \%$ was within the parasitic density of $1-100 / \mu \mathrm{l}$ and the rest $(85.71 \%)$ presented with parasitic density of $1000 / \mu l$ and above. See Table 4.

\section{Discussion}

Malaria is a deadly disease especially in children and, for that reason, it is important to have a prompt and accurate diagnosis of the disease [11]. In this study, the test performance of the three routine malaria diagnostic methods, namely, Rapid Diagnostic Test (RDT), presumptive diagnosis method, and field microscopy technique, was evaluated. The diagnostic accuracy of these methods was measured against a composite and field microscopy as gold standards. In general, the study established that the sensitivity of the three diagnostic methods was very low when evaluated over the composite reference. This is an indication of wider differences or substantial nonoverlap between the test methods in the positive detection of malaria among the study population [6]. In contrast, the sensitivities recorded by the individual test methods were lower than that reported in a similar study in Nigeria by Ojurongbe et al. [7] which had $62.3 \%$ and $77.2 \%$ for RDT and field microscopy, respectively. This difference in recorded sensitivity could be accounted for by the makeup of the composite reference where polymerase chain reaction was inclusive in the previous study but this is not included in this study. Again, whereas this study made use of presumptive diagnosis, the study by Ojurongbe et al. [7] did not. Thus, problems arise in comparison when established methods are used as a composite gold standard without allowing for inherent accuracies for the individual test methods [12].

Diagnosis of malaria has traditionally been based on presumptive diagnosis [4] and its principles are based on nonspecific signs and symptoms like headaches, fever, weakness, dizziness, vomiting, abdominal pains, myalgia, chills, and pruritus [7]. In the current study presumptive diagnosis demonstrated a generally low specificity when compared with the two gold standards: composite (23.55\%) and field microscopy $(25.82 \%)$ as shown in Tables 1 and 2, respectively. These findings were consistent with a previous work which recorded a specificity ranging from 0 to $9 \%$ [13]. Thus, presumptive diagnosis is unable to identify patients without malaria as truly uninfected (false positives). This leads to treating all fevers presumptively as malaria, thereby masking underlying potentially fatal conditions [14]. From the present study, out of 221 people who were clinically diagnosed with malaria, 17 and 24 were positive by field microscopy and RDT, respectively. This shows that about $70 \%$ of patients who were diagnosed as having malaria presumptively turned out to be parasite negative. This massive malaria overdiagnosis according to Reyburn et al. [15] threatens the sustainability of deployment of artemisinin combination treatment, and treatable bacterial diseases are likely to be missed.

After age stratification, the highest sensitivity for the two test methods against the field microscopy technique was recorded among $\leq 5$ years group (Table 2 ). This agrees with the findings of Nkrumah et al. [16] where an increase in the sensitivity of a histidine-rich protein 2 (Pfhrp2) assay was found in children compared to adults. Nkrumah et al. [16] posited that lower immunity and possibly less interference by antibodies among children could be attributable to such outcome. Parasitaemia in older aged groups often remains very low and frequently undetectable by conventional malaria diagnostics (microscopy) and Rapid Diagnostic Test [17].

Rapid Diagnostic Tests (RDTs) based on histidine-rich protein 2 (Pfhrp2) have shown a varied accuracy for malaria infection in field studies, with field microscopy taken as the gold standard $[1,18-20]$. In the present study, RDT reported a sensitivity of $62.5 \%$ and specificity of $92.93 \%$ when compared with field microscopy. This is within the same range as previous studies [21, 22]. However, the sensitivity was lower than the World Health Organization (WHO) recommended minimal standard of $95 \%$ sensitivity for Plasmodium falciparum densities of $100 / \mu \mathrm{L}$ and a specificity of 95\% for an acceptable Rapid Diagnostic Test for malaria [23]. The diagnostic accuracy of RDTs can be affected by several factors such as quality of the products, storage temperature, humidity, and end users' performance [6].

Persistence of Pfhrp2 protein in circulation after parasite clearance contributes to lower specificity level of RDT $[9,19$, 21]. This may explain the observation in the current study where 9 participants on malaria treatment tested positive for RDT with no parasitic detection by field microscopy (false positives). In the case where the parasite density is below the threshold for detection by microscopy (submicroscopic parasitic density), it is still possible for RDT to report positive malaria test [19] or low density parasitaemia might be missed and wrongly classified as negative with microscopy; however the double blind reading of blood films by two experienced microscopists was aimed at reducing the latter scenario [24].

In the current study, out of the 264 patients that RDT confirmed as negative, field microscopy detected 9 of them as positive with 8 patients recording a parasitic density of $\geq 1000$ parasites $/ \mu \mathrm{L}$ (Table 4 ). Studies have reported patients with high levels of parasitaemia that give false negative RDT results due to the deletion of Pfhrp2 antigens or genetic variability in the Pfhrp2 gene in certain Plasmodium falciparum parasites $[25,26]$.

In a study by Abeku et al. [24], false positive error rates declined with increasing age of patients and this was probably a result of acquired immunity in clearing parasite antigen. Contrary to this, the present study noted false positive results by RDT increased with increasing age of patients. According to the manufacturer's manual, internal evaluation was performed on the SD Bioline malaria Ag Pf. 
test which reported a $100 \%$ sensitivity for parasitic count between 101 and 500 parasites $/ \mu \mathrm{L}$ but the present study reported a sensitivity of $75 \%$ for a similar parasitic count of 101-1000 parasites/ $\mu \mathrm{L}$. Manufacturer's specified sensitivity was $100 \%$ for a parasitic count greater than 1000 parasites $/ \mu \mathrm{L}$ whilst this study had a sensitivity of $57.89 \%$ for that same parasitic count. The manufacturer's overall sensitivity when microscopy was used as a reference was greater or equal to $99 \%$ whilst this study reported $62.5 \%$ overall sensitivity.

A patient who reported Plasmodium malariae infection was also positive with the RDT which has been designed to detect only Plasmodium falciparum. This revelation may be due to cross-reactivity [9]. RDT also was positive to a parasite count that was as low as 40 parasites $/ \mu \mathrm{L}$ and this is in contrast to other studies $[6,10,27]$.

The weighted kappa statistic, widely used as a chancecorrected measure for nominal agreement [28], was used to test interrater agreement between microscopy and the other two diagnostic methods (RDT and presumptive diagnosis). There was a moderate level of agreement between RDT (kappa: 0.457) and microscopy. This observation is in accordance with the findings of Ali et al. [29] who reported a moderate agreement between RDT and field microscopy in Cameroon. But the agreement observed was slightly higher compared to that reported by Kilonzo et al. [30] (kappa: 0.354 ) in Tanzania. A recent study in Yemen by Alareqi et al. [31] also reported a kappa of 0.379 for both febrile and afebrile participants and a higher weighted kappa of 0.638 among only febrile patients. Thus RDT could be used for malaria diagnosis in settings where microscopy is not available [32]. Presumptive diagnosis (kappa: 0.007) however had a poor or no agreement with microscopy and thus it is likely to misdiagnose malaria if it is not used alongside a laboratory based diagnostic tool.

The discriminating power of RDT and presumptive diagnosis for the detection of malaria in patients was further investigated by the area under the receiver operative characteristics (ROC) as seen in Table 3. It was found that RDT (area under the diagnostic curve (AUC: 0.697)) was more effective in predicting malaria infection; however in the work of Djimde et al. [33] in Mali, RDT recorded a higher area under the ROC curve (0.97). The presumptive diagnosis method had an area under the ROC curve of -0.506 , which implies that it is a worthless test for malaria diagnosis.

Youden index was carried out to measure the medical usefulness of the diagnostic methods for malaria detection. RDT proved to be a useful diagnostic test (Youden: 0.395) as compared to presumptive diagnosis (Youden: 0.013). The performance of the RDT agrees with the findings of Samadoulougou et al. [34] in Burkina Faso who reported an overall Youden of 0.40 . This shows that presumptive diagnosis is less effective and an unreliable diagnostic tool for malaria diagnosis as a standalone test.

\section{Conclusion}

Based on the results of this study, none of the test methods evaluated over the gold standard (field microscopy technique) achieved the WHO recommended diagnostic sensitivity and specificity. However, the RDT achieved an acceptable agreement with the gold standard. Factors such as medication and age also played a role in influencing the test performance of the various diagnostic methods. Diagnosis of malaria by field microscopy should still be the gold standard although it requires a level of expertise. In settings where microscopy is not available, however, RDTs must be preferred as a confirmation of presumptive diagnosis.

\section{Ethical Approval}

Approval for this study was granted by the authorities of the facility. The study was anonymous and nonlinked; participation was voluntary and all patients or their parents consented to participation in this study. All patients in this study had been referred to the laboratory as part of the clinical care they were receiving from the hospital. No patient was denied the appropriate care for declining to participate in this study.

\section{Competing Interests}

Authors have declared that no competing interests exist.

\section{Authors' Contributions}

This work was carried out in collaboration between all authors. J. Osei-Yeboah, G. K. Norgbe, S. Y. Lokpo, M. K. Kinansua, L. Nettey, and E. A. Allotey conceptualized and designed the study. J. Osei-Yeboah, S. Y. Lokpo, M. K. Kinansua, and L. Nettey recruited participants and generated the data. J. Osei-Yeboah, S. Y. Lokpo, M. K. Kinansua, and E. A. Allotey analyzed the data. J. Osei-Yeboah, G. K. Norgbe, S. Y. Lokpo, M. K. Kinansua, and E. A. Allotey drafted the manuscript. J. Osei-Yeboah, G. K. Norgbe, S. Y. Lokpo, M. K. Kinansua, L. Nettey, and E. A. Allotey reviewed the manuscript for intellectual content and each author approved the final manuscript.

\section{Acknowledgments}

The authors want to thank the management of the Ho Municipal Hospital for giving them the permission to carry out this study in their facility. Their sincere gratitude goes to all biomedical scientists at the Ho Municipal Hospital, for without their participation this project would not have come into fruition.

\section{References}

[1] B. B. Andrade, A. Reis-Filho, A. M. Barros et al., "Research Towards a precise test for malaria diagnosis in the Brazilian Amazon: comparison among field microscopy, a Rapid Diagnostic Test, nested PCR, and a computational expert system based on artificial neural networks," 2010.

[2] A. Abdul-Aziz, E. Harris, and L. Munyakazi, "Risk factors in malaria mortality among children in Northern Ghana: a case study at the tamale teaching hospital," International Journal of Business and Social Research, vol. 2, no. 5, pp. 35-45, 2012. 
[3] N. Tangpukdee, C. Duangdee, P. Wilairatana, and S. Krudsood, "Malaria diagnosis: a brief review," The Korean Journal of Parasitology, vol. 47, no. 2, pp. 93-102, 2009.

[4] B. S. C. Uzochukwu, E. N. Obikeze, O. E. Onwujekwe, C. A. Onoka, and U. K. Griffiths, "Cost-effectiveness analysis of rapid diagnostic test, microscopy and syndromic approach in the diagnosis of malaria in Nigeria: implications for scaling-up deployment of ACT," Malaria Journal, vol. 8, no. 1, article 265, 2009.

[5] A. Björkman and A. Mårtensson, "Risks and benefits of targeted malaria treatment based on rapid diagnostic test results," Clinical Infectious Diseases, vol. 51, no. 5, pp. 512-514, 2010.

[6] T. Endeshaw, T. Gebre, J. Ngondi et al., "Evaluation of light microscopy and rapid diagnostic test for the detection of malaria under operational field conditions: A household survey in Ethiopia," Malaria Journal, vol. 7, no. 1, article 118, 2008.

[7] O. Ojurongbe, O. O. Adegbosin, S. S. Taiwo et al., "Assessment of clinical diagnosis, microscopy, rapid diagnostic tests, and polymerase chain reaction in the diagnosis of Plasmodium falciparum in Nigeria," Malaria Research and Treatment, vol. 2013, Article ID 308069, 5 pages, 2013.

[8] H. Hopkins, L. Bebell, W. Kambale, C. Dokomajilar, P. J. Rosenthal, and G. Dorsey, "Rapid diagnostic tests for malaria at sites of varying transmission intensity in Uganda," The Journal of Infectious Diseases, vol. 197, no. 4, pp. 510-518, 2008.

[9] C. Wongsrichanalai, M. J. Barcus, S. Muth, A. Sutamihardja, and W. H. Wernsdorfer, "A review of malaria diagnostic tools: microscopy and Rapid Diagnostic Test (RDT)," The American Journal of Tropical Medicine and Hygiene, vol. 77, no. 6, supplement, pp. 119-127, 2007.

[10] E. G. Long, "Requirements for diagnosis of malaria at different levels of the laboratory network in Africa," American Journal of Clinical Pathology, vol. 131, no. 6, pp. 858-860, 2009.

[11] P. Mens, N. Spieker, S. Omar, M. Heijnen, H. Schallig, and P. A. Kager, "Is molecular biology the best alternative for diagnosis of malaria to microscopy? A comparison between microscopy, antigen detection and molecular tests in rural Kenya and urban Tanzania," Tropical Medicine and International Health, vol. 12, no. 2, pp. 238-244, 2007.

[12] D. R. Bell, D. W. Wilson, and L. B. Martin, "False-positive results of a Plasmodium falciparum histidine-rich protein 2detecting malaria rapid diagnostic test due to high sensitivity in a community with fluctuating low parasite density," The American Journal of Tropical Medicine and Hygiene, vol. 73, no. 1, pp. 199-203, 2005.

[13] J. Chipeta, S. Mharakurwa, O. Thuma, and N. Kumar, "A synopsis of current malaria diagnosis trends," Medical Journal of Zambia, vol. 36, no. 2, 2009.

[14] M. Amexo, R. Tolhurst, G. Barnish, and I. Bates, "Malaria misdiagnosis: effects on the poor and vulnerable," Rapid Review, vol. 364, 2004.

[15] H. Reyburn, H. Mbakilwa, R. Mwangi et al., "Rapid diagnostic tests compared with malaria microscopy for guiding outpatient treatment of febrile illness in Tanzania: randomised trial," British Medical Journal, vol. 334, no. 7590, pp. 403-406, 2007.

[16] B. Nkrumah, S. E. K. Acquah, L. Ibrahim et al., "Comparative evaluation of two rapid field tests for malaria diagnosis: Partec Rapid Malaria Test ${ }^{\circledR}$ and Binax Now ${ }^{\circledR}$ Malaria Rapid Diagnostic Test," BMC Infectious Diseases, vol. 11, no. 1, article 143, pp. 1-8, 2011.
[17] L. Golassa, F. N. Baliraine, N. Enweji, B. Erko, G. Swedberg, and A. Aseffa, "Microscopic and molecular evidence of the presence of asymptomatic Plasmodium falciparum and Plasmodium vivax infections in an area with low, seasonal and unstable malaria transmission in Ethiopia," BMC Infectious Diseases, vol. 15, article 310, 2015.

[18] Z. Bisoffi, S. B. Sirima, J. Menten et al., "Accuracy of a rapid diagnostic test on the diagnosis of malaria infection and of malaria-attributable fever during low and high transmission season in Burkina Faso," Malaria Journal, vol. 9, no. 1, article 192, 2010.

[19] D. R. Bell, D. W. Wilson, and L. B. Martin, "False-positive results of a Plasmodium falciparum histidine-rich protein 2detecting malaria rapid diagnostic test due to high sensitivity in a community with fluctuating low parasite density," The American Journal of Tropical Medicine and Hygiene, vol. 73, no. 1, pp. 199-203, 2005.

[20] V. Batwala, P. Magnussen, and F. Nuwaha, "Are rapid diagnostic tests more accurate in diagnosis of Plasmodium falciparum malaria compared to microscopy at rural health centres?" Malaria Journal, vol. 9, no. 1, article 349, 2010.

[21] M. L. Mcmorrow, M. I. Masanja, S. M. K. Abdulla, E. Kahigwa, and S. P. Kachur, "challenges in routine implementation and quality control of Rapid Diagnostic Tests for malaria-Rufiji district," The American Society of Tropical Medicine and Hygiene, vol. 79, no. 3, pp. 385-390, 2008.

[22] D. O. Acheampong, M. G. Appiah, L. K. Boamponsem, J. N. Boampong, and R. Afoakwa, "the efficacy of Rapid Diagnostic Test(rdt) in diagnosing plasmodium falciparum in some selected health facilities in the capecoast metropolis of Ghana," Advances in Applied Science Research, vol. 2, no. 4, pp. 348-356, 2011.

[23] C. K. Murray, R. A. Gasser Jr., A. J. Magill, and R. S. Miller, "Update on rapid diagnostic testing for malaria," Clinical Microbiology Reviews, vol. 21, no. 1, pp. 97-110, 2008.

[24] T. A. Abeku, M. Kristan, C. Jones et al., "Determinants of the accuracy of rapid diagnostic tests in malaria case management: evidence from low and moderate transmission settings in the East African highlands," Malaria Journal, vol. 7, no. 1, article 202, 2008.

[25] J. C. Mouatcho and J. P. D. Goldring, "Malaria rapid diagnostic tests: challenges and prospects," Journal of Medical Microbiology, vol. 62, no. 10, pp. 1491-1505, 2013.

[26] M. L. Wilson, laboratory Diagnosis of Malaria, Archives of Pathology \& Laboratory Medicine, 2013.

[27] L. M. Singer, R. D. Newman, A. Diarra et al., "Evaluation of a malaria rapid diagnostic test for assessing the burden of malaria during pregnancy," The American Journal of Tropical Medicine and Hygiene, vol. 70, no. 5, pp. 481-485, 2004.

[28] M. A. Billo, M. Diakité, A. Dolo et al., "Inter-observer agreement according to malaria parasite density," Malaria Journal, vol. 12, no. 1, article 335, pp. 1-6, 2013.

[29] I. M. Ali, J. D. Bigoga, D. A. Forsah et al., "Field evaluation of the 22 rapid diagnostic tests for community management of malaria with artemisinin combination therapy in Cameroon," Malaria Journal, vol. 15, no. 1, article 31, pp. 1-7, 2016.

[30] S. B. Kilonzo, E. Kamugisha, J. A. Downs et al., "Malaria among adult inpatients in two Tanzanian referral hospitals: a prospective study," Acta Tropica, vol. 134, no. 1, pp. 95-100, 2014.

[31] L. M. Q. Alareqi, M. A. K. Mahdy, Y.-L. Lau et al., "Field evaluation of a PfHRP-2/pLDH rapid diagnostic test and light 
microscopy for diagnosis and screening of falciparum malaria during the peak seasonal transmission in an endemic area in Yemen," Malaria Journal, vol. 15, no. 1, article 49, 9 pages, 2016.

[32] E. K. Ansah, S. Narh-Bana, M. Epokor et al., "Rapid testing for malaria in settings where microscopy is available and peripheral clinics where only presumptive treatment is available: a randomised controlled trial in Ghana," British Medical Journal, vol. 340, article c930, 2010.

[33] A. A. Djimde, H. Maiga, I. Sagara et al., "Field assessment of SD bioline malaria species antigen detection by rapid diagnostic tests in Mali," Journal of Parasitology and Vector Biology, vol. 8, no. 1, pp. 1-9, 2016.

[34] S. Samadoulougou, F. Kirakoya-Samadoulougou, S. Sarrassat et al., "Paracheck ${ }^{\circledR}$ rapid diagnostic test for detecting malaria infection in under five children: a population-based survey in Burkina Faso," Malaria Journal, vol. 13, no. 1, article 101, 2014. 

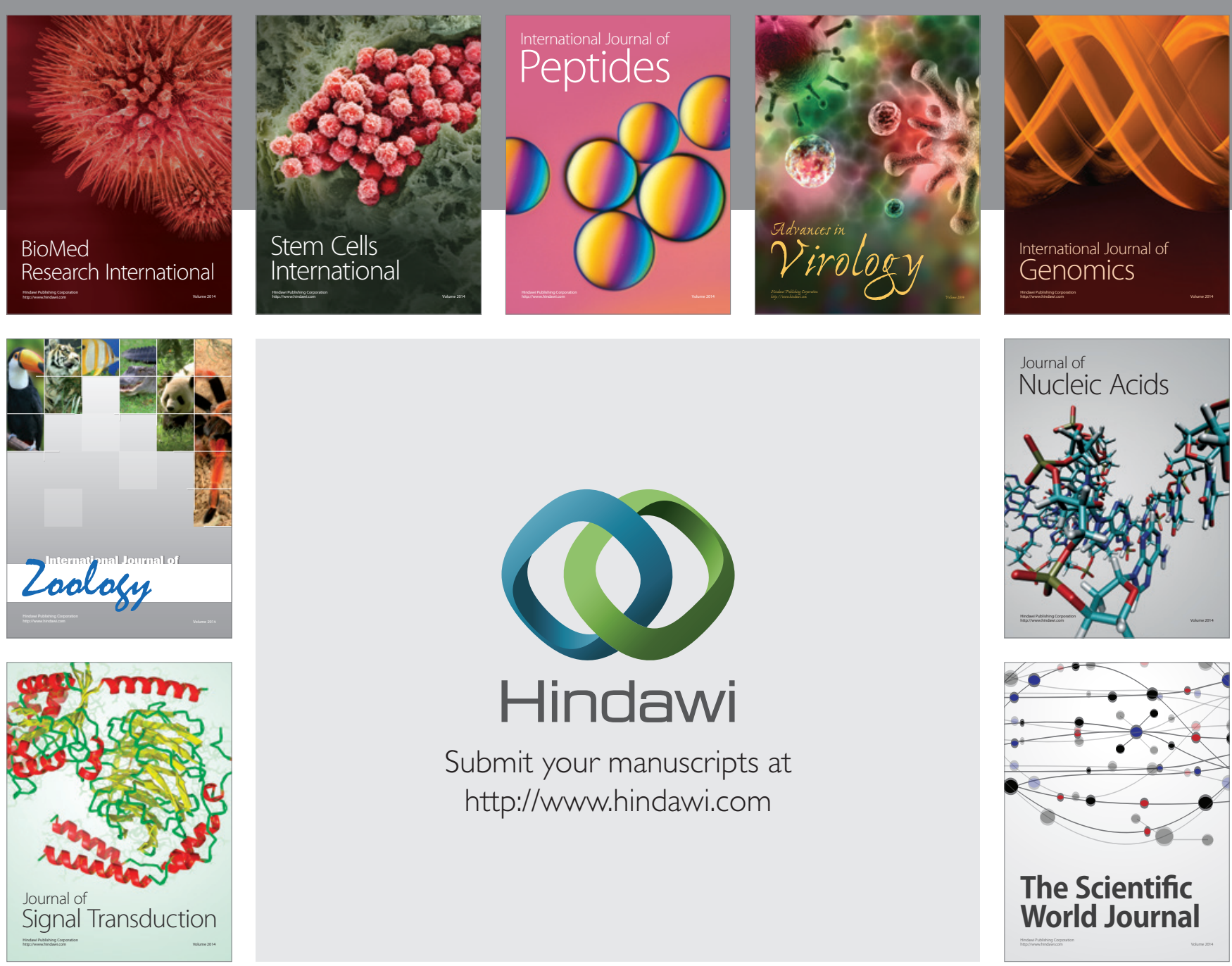

Submit your manuscripts at

http://www.hindawi.com
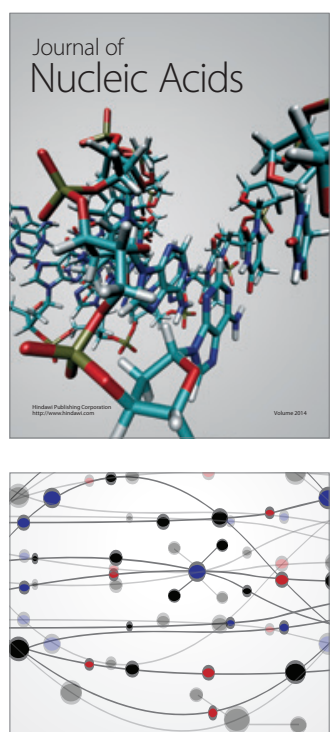

The Scientific World Journal
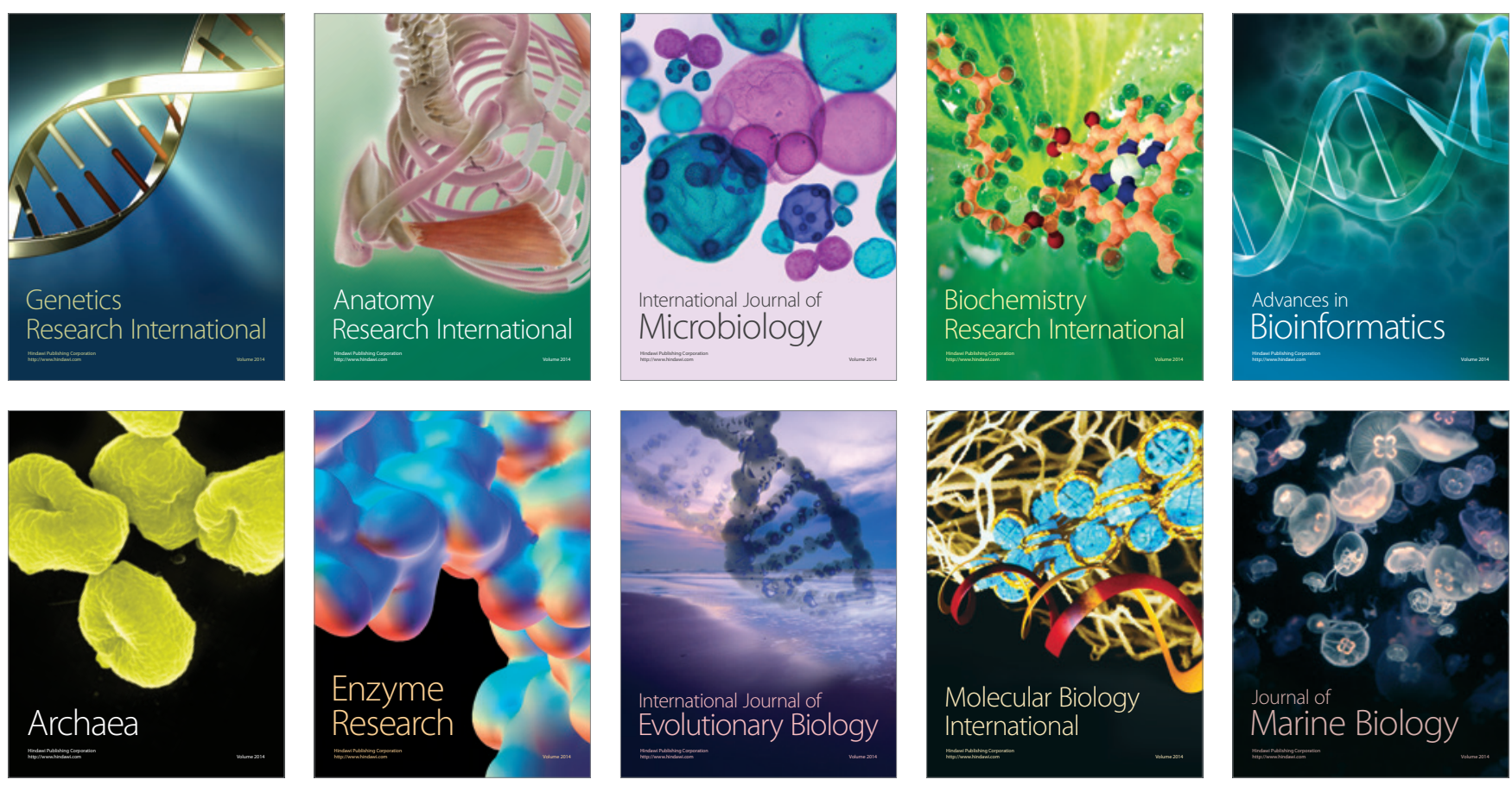